\title{
Search for a quick getaway from Mars
}

Sir - I was heartened to see your article on Carlo Rubbia's proposed propulsion system to send humans to Mars (Nature 397, 374; 1999). I emphatically agree with Rubbia that fast missions will be required to reduce to manageable levels the dose of galactic cosmic rays to which the crew would be exposed. Calculations at the Los Alamos National Laboratory in New Mexico indicate that the crew could receive their entire allowable lifetime dose of $200 \mathrm{cSv}$ during a three-year mission. More than half of this dose would come from radiation on the surface of the planet.

Rubbia is also correct in stating that a new, advanced, high-performance propulsion system would allow fast missions with return trips of about one year. Such a mission is well within human experience -500 days trapped in a pup tent on the surface of an unknown world is not.

I must disagree with the article and

Rubbia with respect to the stated performance of the NERVA nuclear rocket. The US space agency NASA is currently trying to use chemical rockets, such as those on the Space Shuttle, in its mission studies, which would allow round-trip missions of three years. During the 1985 Manned Mars Mission Study by the Los Alamos laboratory and NASA, the impact of using a NERVA rocket was reinvestigated. Los Alamos also proposed redevelopment of the NERVA engine during the Space Exploration Initiative. NERVA was tested by Los Alamos in the 1960s, having a specific impulse of 850 seconds. Such an engine would allow a Mars mission of 434 days for the same mass in low Earth orbit that the chemical engines will require. This is proven technology.
Los Alamos has also been studying a concept, the gas-core nuclear rocket, that would have a specific impulse equal to the concept of Rubbia, 3,000 seconds. Such an engine would enable a nine-month mission to Mars. In addition, the performance of the rocket allows the spaceship to carry shielding against cosmic rays. Estimates show the dose to the crew would be reduced by more than a factor of ten.

Rubbia's concept is as viable as the gascore rocket concept. To send humans to Mars will require something with the performance of these engines. I eagerly await the results of the design analysis of the Rubbia concept with regard to engine mass, radiator mass, power requirements and thermal loading.

Steven D. Howe

Los Alamos National Laboratory, New Mexico, USA

\section{Sartorial simplicity is}

\section{knot what it seems}

Sir-As a former denizen of the British boarding-school system, I was delighted to find that simpler and mathematically rigorous methods exist for knotting a tie (Nature 398, 31-32; 1999).

I wondered, however, whether experimental testing might reveal unexpected flaws in the modelling process.

I began with the three-move reversestart knot, which is one move shorter than the four-in-hand knot I learned as a schoolboy. All was well. Until... as I went to remove the experimental apparatus (my old-boy tie), I discovered to my horror that the 'simpler' knot left me with an overhand loop. The four-in-hand (and all knots with an even number of moves, including the Windsor and half-Windsor) leaves no such lingering trace requiring further engineering solutions.

I have reached three major conclusions from my experiment. (1) I shall generally stick with the method I learned in my Dickensian past, unless (2) I am late for an appointment requiring a tie, and the fraction of a second gained at the outset outweighs the delayed cost of undoing an overhand loop at the end of the appointment. And (3) a model is rarely complete until it has been field-tested in a variety of contexts.

Incidentally, many inhabitants in this part of North America have circumvented the entire suite of theoretical and applied problems entailed in knot tying by adopting the bolo tie.

\section{David B. McDonald}

Department of Zoology and Physiology, University of Wyoming, Laramie, Wyoming 82071-3166, USA

Sir - I have spent some time in front of a mirror studying the conclusions of the Fink-Mao analysis of tie knots. As my profession of lexicography involves close attention to terminology, I was interested to note the authors' restraint in not proposing names for the unnamed knots.

The first knot in the table, $\{3,1\}$, which is even simpler than the common 'four-inhand' $\{4,1\}$, is presumably a 'three-inhand'. The 'inside-out' knot $\{7,2\}$, after its introductory move, in fact consists of the same moves as the half-Windsor $\{6,2\}$, but inverted left to right. It might be referred to as the 'inverse half-Windsor'. The more complex knot $\{9,3\}$ bears the same relationship to the Windsor, and so might be called the 'inverse Windsor'.

The knot $\{7,3\}$ concludes with the same winding sequence as the half-Windsor, but begins with an 'LC' move reminiscent of the full Windsor, and reproducing the beginning of the Pratt knot $\{5,2\}$. I suggest it must be either a 'three-quarter-Windsor' or a 'Pratt-Windsor'.

That leaves the curious $\{8,2\}$, a superficially elegant knot which (as an Oxford man) I suggest calling the 'Cambridge', and the monumental $\{9,4\}$, for which the name 'Cavendish' presents itself, in honour of the originating institution.

Jeremy H. Marshall

54 North Hinksey Village, Oxford OX2 ONA, UK

\section{Grant agencies must be seen to be fair}

Sir - Each year the International Agency for Research on Cancer (IARC) in Lyons, France, announces a competition for training fellowships. As a rejected candidate in last year's round, I would like to draw attention to what I consider to be a nontransparent system of selection, which fails in its responsibility to give feedback to young scientists trying to establish a career.

I was given only seven days notice of an interview which was conducted by only one person. Yet, despite this short notice, I had to wait three months before being told by fax - that my application was unsuccessful.

I immediately asked the IARC for the reason for my rejection. I was told that it was against agency policy to give such information. I then asked for more precise information about the list of applicants, the scoring each received and, again, the reason for my failure to be shortlisted. After some delay I was told that this information was confidential.

Obtaining a research fellowship is a very important step in the career of a young researcher. I consider it fair that unsuccessful applicants should be allowed to understand the reasons for their exclusion and the evaluation criteria used. And interviews should be conducted more openly. IARC, and all grant-giving agencies, should employ more transparent practices. Stefano Parodi

Ligurian Cancer Registry, National Cancer Institute, Largo R. Benzi 10, 16132 Genova, Italy 